\title{
EVOLUTION OF DAMAGE ACCUMULATION IN LOW-CARBON STEEL IN TENSION CONDITION
}

\author{
M.R. TYUTIN ${ }^{1}$, L.R. BOTVINA ${ }^{1}$, N.A. ZHARKOVA ${ }^{1}$, T.B. PETERSEN ${ }^{2}$, J.A. \\ $\mathrm{HUDSON}^{3}$ \\ ${ }^{1}$ A.A. Baikov Institute of Metallurgy and Materials Science, Moscow, Russia \\ ${ }^{2}$ PRC Kurchatov Institute, Moscow, Russia \\ ${ }^{3}$ Cambridge University, UK
}

\begin{abstract}
Stages of damage accumulation process in notched specimens from low-carbon steel at tension have been studied by means of replicas, acoustic emission and ultrasound attenuation methods. The shape and size of plastic deformation zones at a pre-existing crack tip, microcrack length and density in the zones have been estimated and cumulative distributions of microcracks by their size have been plotted. Using acoustic emission method, number and amplitude of acoustic emission signals characterizing damage accumulation process on various stages of loading and also location of the signals on the specimen surface have been estimated and cumulative amplitude distributions of the signals have been plotted. It has been established that cumulative distributions of microcracks and acoustic emission signals on initial stage of damage accumulation are well described by an exponential function which is replaced by a power law one at a load close to the maximum value. At this stage of loading, density of microcracks and acoustic emission signals approaches the limited value, crack coalescence leading to the main crack formation begins and ultrasound attenuation coefficient sharply increases. The results obtained are compared to the regularities of fault accumulation in the earth crust.
\end{abstract}

\section{INTRODUCTION}

The process of fracture is multistage and involves the stages of nucleation, accumulation and growth of defects; their interaction; formation of the main crack; and the final fracture. Among the stages listed, the last stage of localized fracture described in the context of the fracture mechanics is the most extensively studied. The stage of multiple or distributed fracture, when the accumulation and growth of different-scale defects occurs, is the least understood because it typically requires the use of sophisticated techniques for the evaluation of defects of various kinds. However, this may be labour well spent, since an analysis of the damage accumulation process in the materials belonging to different classes and scales has many common features allowing considering this process in terms of the theory of critical events. Therefore the main goal of this paper is establishing common peculiarities preceding critical event (main crack formation or fracture) observed by various methods and characterizing mechanical and physical behavior of material.

\section{ESTIMATION OF ULTRASOUND ATTENUATION}

Ultrasound attenuation coefficients were estimated at various stages of loading and after loading (Botvina et al [1]). Measurements have been carried out in the plastic deformation zone near a crack tip at frequency of $5 \mathrm{MHz}$ or under specimen fracture surface at range of frequencies from 2 to $20 \mathrm{MHz}$. The ultrasound attenuation coefficient increased with displacement and decreased with decreasing grain size of specimen material (Fig. 1). In Fig. 2, a dependence of attenuation 
coefficient at various stages of loading on microcrack density is presented. The microcrack density has been evaluated using relation (1) from studies of (Peacock et al.[2, 3] ):

$$
\mathrm{CD}=\frac{\mathrm{N} \cdot 1^{3}}{\mathrm{~S} \cdot \mathrm{t}},
$$

where $\mathrm{N}$ is the number of cracks, 1 is the mean length of cracks, $\mathrm{S}$ is the surface area, $\mathrm{t}$ is the specimen thickness.

As can be seen from Fig. 2, the attenuation coefficient is linearly related to the microcrack density estimated at different displacements corresponding to various loading stages. Similar relationship between the attenuation coefficient and a crack density in specimens from marble has been obtained in [Peacock et al. [2]) where the accordance of experimental data on ultrasound attenuation with Hudson's theory (Hudson [4]) has been verified (see also Piau [5] and Peacock \& Hudson [6]). Further verification of this relationship has been provided by Saenger et al. [7], using three-dimensional numerical studies.

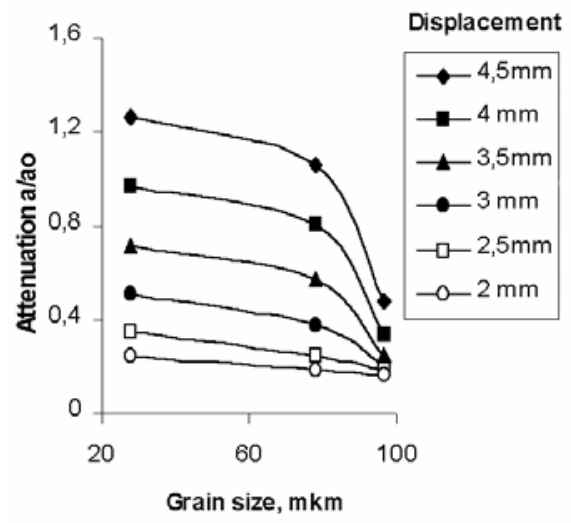

(a)

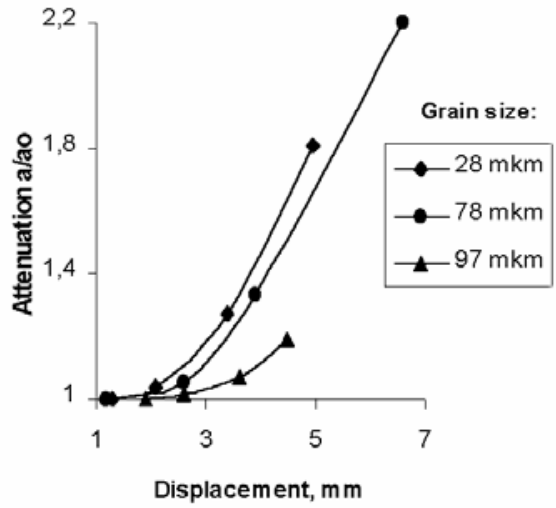

(b)

Fig. 1. Dependence of a relative ultrasound attenuation coefficient on a grain size and deformation of notched specimen from low-carbon steel

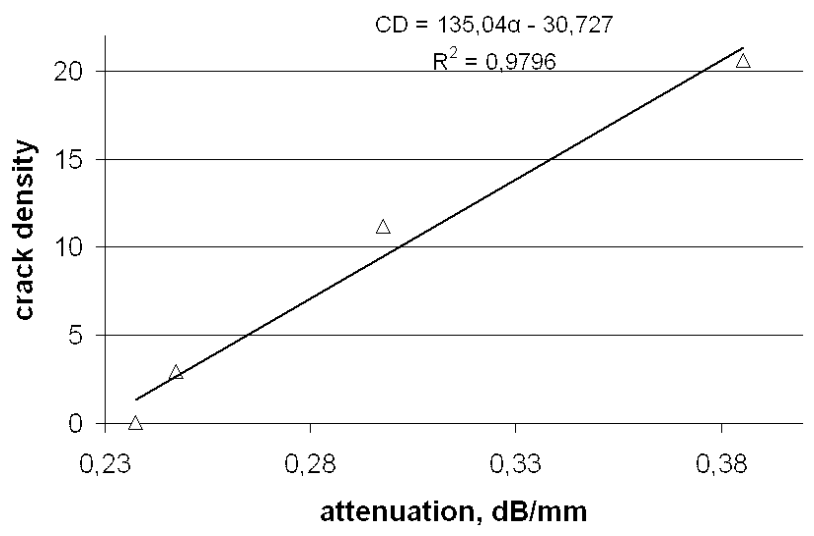

Fig. 2. Dependence of microcrack density at various stages of tension on ultrasound attenuation 


\section{ACOUSTIC EMISSION DATA}

In Fig.3 ( $a, b, c)$, the maps of acoustic emission signals location at various loading stages together with the changes of plastic zones that caused the acoustic activity are presented (Botvina et al.[1]). It can be seen that acoustic emission data correspond well to the increase in plastic zone which contain branches of localized deformation on initial stages (Fig. 3, d, e). With further increase in load, these branches merge and the second highly deformed plastic zone appears near crack tip of the newly formed main crack (Fig. 3, f).

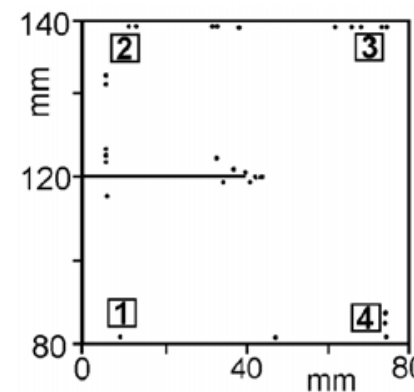

(a)

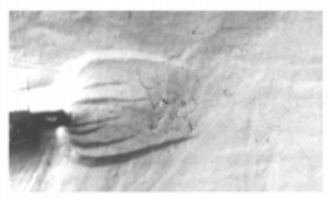

(d), $\times 3.5$

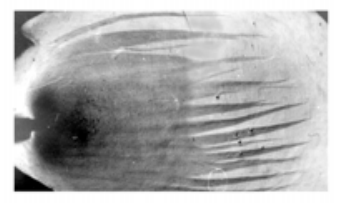

(e), $\times 3.5$

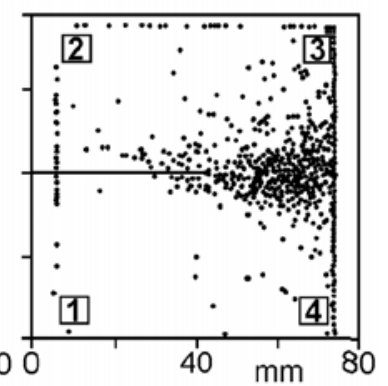

(c)

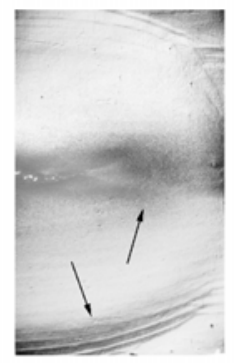

(f), $\times 2.5$

Fig. 3.Results of acoustic emission signal location at various stages of tension of notched specimens $(\mathrm{a}, \mathrm{b}, \mathrm{c})$ and corresponding plastic zones obtained by means of replicas method (d, e, f)

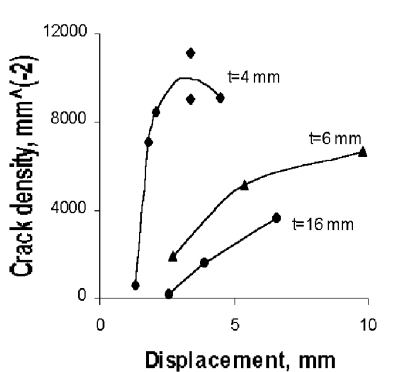

(a)

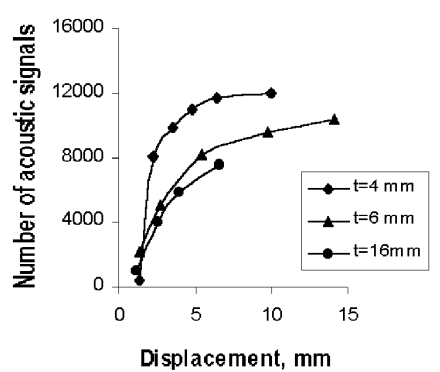

(b)

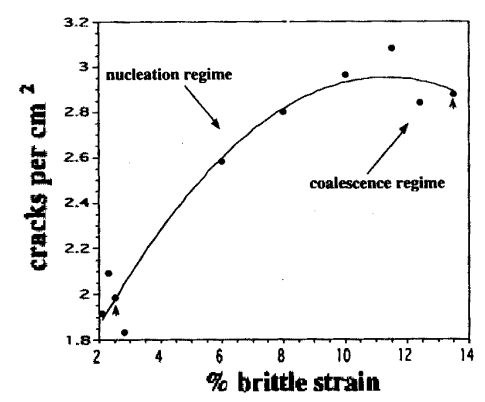

(c)

Fig. 4. Dependences of microcrack density (a) and number of acoustic emission signals (b) on deformation [1] and development of population of faults in model of Spyropoulos (Scholz [8]) 
A good correlation between the acoustic emission data and microcrack density within plastic deformation zones was revealed (Fig. 3). From Fig. 3 it follows that both parameters, the microcrack density and the corresponding number of acoustic emission signals, increase and reach limiting values with increasing displacement. The higher limited values of the characteristics correspond to the lower specimen thickness (Fig. 4,b). It means probably that the rate of microcrack initiation decreases as a result of their coalescence near the maximum tensile load, and such a decrease depends on the local stress state at the tip of a crack forming in the notched specimen near the maximum load. Acoustic emission captures this process well.

Similar dependence of a crack density on a displacement is used in the model describing crack population in brittle rock materials (Fig. 4, c) (Scholz [8]).

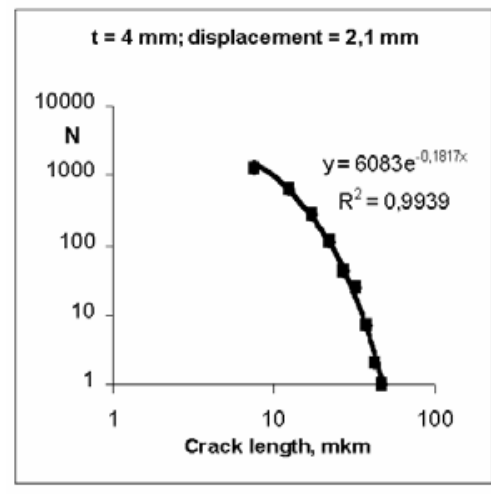

(a)

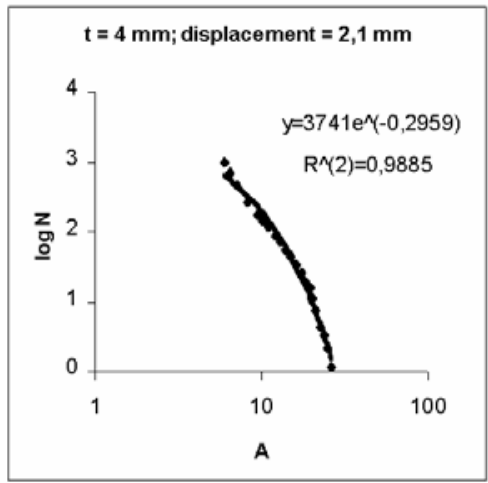

(c)

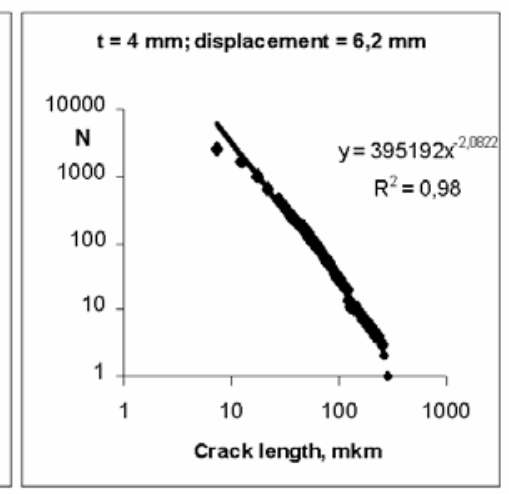

(b)

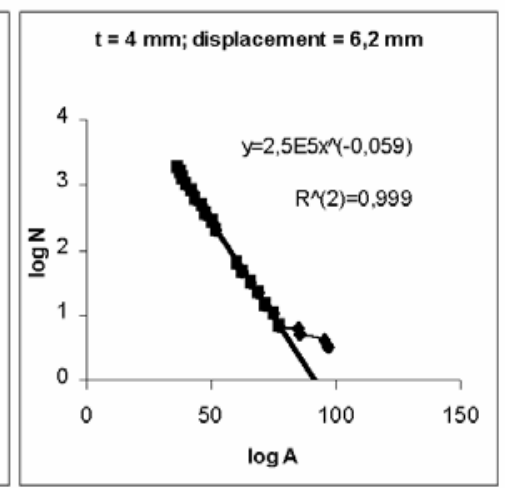

(d)

Fig. 5. Cumulative distributions of microcracks (a, b) and acoustic emission signals (c, d) on an initial stage of plastic deformation $(a, c)$ and near the maximum load $(b, c)$

The coalescence of cracks leads to a change in the function describing cumulative distributions of microcracks by their size and acoustic emission signals by their amplitude (Fig. 5). It was found that both type of curves, different a nature, manifest the same changes with load increasing. At initial stages of loading, the curves are best fitted by an exponential function that is replaced by the power law one near the maximum load of fracture. This transition occurs at lower displacement and loads when specimens of larger thickness are used. At an intermediate stage of loading, the 
cumulative curves may be described both by an exponential function or a power law function with the same correlation coefficient.

Thus, the shape of the cumulative distributions of microcracks and acoustic emission signals is defined, probably, by the local stress state at a crack tip in a specimen and a "distance" to a critical event of the process, namely, to the formation of the main crack at the maximum fracture load. As a "distance" to a critical event one may use the difference $\left(\varepsilon_{c}-\varepsilon\right)$ of the critical deformation at the maximum load $\left(\varepsilon_{\mathrm{c}}\right)$ and the current deformation $(\varepsilon)$.

Considering the fracture process in terms of the phase transition theory, this difference may serve as the order parameter of a phase transition during fracture. It has been established that a decrease in this parameter leads to the reduction in the exponent of the cumulative distribution curves characterising the damage accumulation process before the main crack formation.

Similar change of the types of cumulative distributions has been found during studying the natural faults in the oceanic or earth crust. So, the authors of (Cowie et al. [9]) showed that cumulative fault distribution in the oceanic crust is well fitted by an exponential function, but this function becomes the power law one in the zone of active faults in the earth crust. It shows that the regularities of fault development in the earth crust, i.e. on a global scale level, are probably similar to those observed at studying microcrack accumulation in metallic specimens.

The appearance of power law dependence upon reaching of a critical state agrees with the theory of phase transitions according to which the power law function describes phenomena and processes occurring near the critical event. However, many researches report the other sense of change in the functional dependence of the cumulative crack (or fault) distributions. So, in (Scholz [8]) it has been reported that the power law function describes cumulative distribution at the initial stage of fracture development, when the process of initiation and development of individual faults dominates. With increasing fault density, the fault interaction begins, their number decreases, and cumulative distribution becomes a power law one. These differences in the order of change in the functions describing cumulative crack distributions observed in specimens and natural faults may be connected to the difficulties of determining the stage of maximum damage accumulation under natural conditions.

Further investigations must bring to light the order of change in the functions describing the cumulative crack distributions plotted on various scales and corresponding to materials with different structure and strength.

\section{ACKNOWLEDGMENTS}

This work was partially supported by the INTAS (project N 01-5-748) and by the Russian Foundation for Basic Research (project N 02-05-65231-a).

\section{REFERENCES}

[1] Botvina L.R., Zharkova N.A., Tyutin M.R., Petersen T.B., Budueva V.G. Diagnostics of damage accumulation in low carbon steel, Metals, 2004, (in press).

[2] Peacock S., McCann C., Sothcott J. and Astin T.R., Experimental measurements of seismic attenuation in microfractured sedimentary rock. Geophysics, 59, No. 9, 1342-1351, 1994.

[3] Peacock S., McCann C., Sothcott J. and Astin T.R., Seismic velocities in fractured rocks: an experimental verification of Hudson's theory. Geophysical Prosp., 42, 27-80, 1994.

[4] Hudson J.A., Overall properties of a cracked solid, Math. Proc. of the Cambridge Phil. Soc., 88, 371-384, 1980.

[5] Piau, M. Attenuation of a plane compressional wave by a random distribution of thin circular cracks. Int. J. Eng. Sci. 17, 151-167, 1979.

[6] Peacock, S., Hudson, J.A. Seismic properties of rocks with distributions of small cracks. Geophys. J. Int. 102, 471-484, 1990. 
[7] Saenger E.H., Kruger O.S., Shapiro S.A. Effective elastic properties of randomly fractured soils: 3D numerical experiments.Geophys. Prosp. 52, 183-195, 2004.

[8] Scholz C.H., Earthquakes and Faulting, Cambridge Univ. Press, 2002.

[9] Cowie P.A., Malinverno A., Ryan W.B.F., Edwards M.N., Quantitative fault studies on the East Pacific Rise: a comparison of sonar imaging techniques. J. of Geoph. Res., 99 1520515218, 1994. 